\title{
Recent advances in ion sensing with conducting polymers
}

\author{
Vithyasaahar Sethumadhavan', Sam Rudd ${ }^{1}$, Eliza Switalska ${ }^{1}$, Kamil Zuber $^{1}$, Peter Teasdale ${ }^{2}$ and Drew Evans ${ }^{1 *}$ (i)
}

\begin{abstract}
Ions are present throughout our environment-from biological systems to agriculture and beyond. Many important processes and mechanisms are driven by their presence and their relative concentration. In order to study, understand and/or control these, it is important to know what ions are present and in what concentration-highlighting the importance of ion sensing. Materials that show specific ion interaction with a commensurate change in measurable properties are the key components of ion sensing. One such type are conducting polymers. Conducting polymers are referred to as 'active' because they show observable changes in their electrical and optical (and other) properties in response to changing levels of doping with ions. For example, p-type conducting polymers such as poly(3,4ethylenedioxythiophene) and polypyrrole, can transition from semi-conducting to metallic in response to increasing levels of anions inserted into their structure. Under certain circumstances, conducting polymers also interact with cations - showing their utility in sensing. Herein, recent advances in conducting polymers will be reviewed in the context of sensing ions. The main scope of this review is to critically evaluate our current understanding of ion interactions with conducting polymers and explore how these novel materials can contribute to improving our ion-sensing capabilities.
\end{abstract}

Keywords: Conducting polymers, lons, Sensing

\section{Introduction}

Many processes and mechanisms found in nature rely on ions. For example, within the body, the relative concentration of particular ions regulate the function of cells. Similarly, micronutrient ions within soil water may be available for uptake by plants to facilitate growth. Examples such as these highlight the desire to determine what ions are present in a system, and in what concentration. Ion sensors offer a promising way to continuously monitor these species, typically in aqueous media [1]. For example, ion sensors are applied in the field of agriculture for plant growth, healthcare for diagnostics and industrial sectors (e.g. water treatment for potable supply), as shown in Fig. 1. It is common in such applications for a range of different ions to be present, both cations and anions, of varying chemistry and concentrations. This

\footnotetext{
*Correspondence: drew.evans@unisa.edu.au

${ }^{1}$ Future Industries Institute, University of South Australia, Mawson Lakes, SA 5095, Australia

Full list of author information is available at the end of the article
}

adds complexity due to the possible competitive processes that inhibit the sensors' ability to detect the ion of interest.

Selective interactions between ions and materials are necessary but insufficient to developing ion-sensing capabilities. The interaction must also produce quantifiable and reversible property changes over a short time period. If the change in property can be related to the presence and concentration of an ion of interest, then an effective ion sensor may be feasible.

One class of materials that show promise in this regard is conducting polymers. These materials have properties that depend on the type and number of ions that reside within (dope) the polymer. The change in doping level leads to changes in the oxidation level of the polymer-yielding observable (and measurable) changes in the polymer properties. Conducting polymers possess a diverse range of properties that make them desirable for a range of applications beyond just sensing (summarised in Table 1).

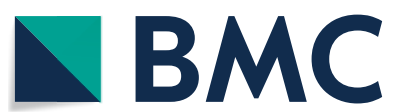

C The Author(s) 2019. This article is distributed under the terms of the Creative Commons Attribution 4.0 International License (http://creativecommons.org/licenses/by/4.0/), which permits unrestricted use, distribution, and reproduction in any medium, provided you give appropriate credit to the original author(s) and the source, provide a link to the Creative Commons license, and indicate if changes were made. The Creative Commons Public Domain Dedication waiver (http://creativecommons.org/ publicdomain/zero/1.0/) applies to the data made available in this article, unless otherwise stated. 


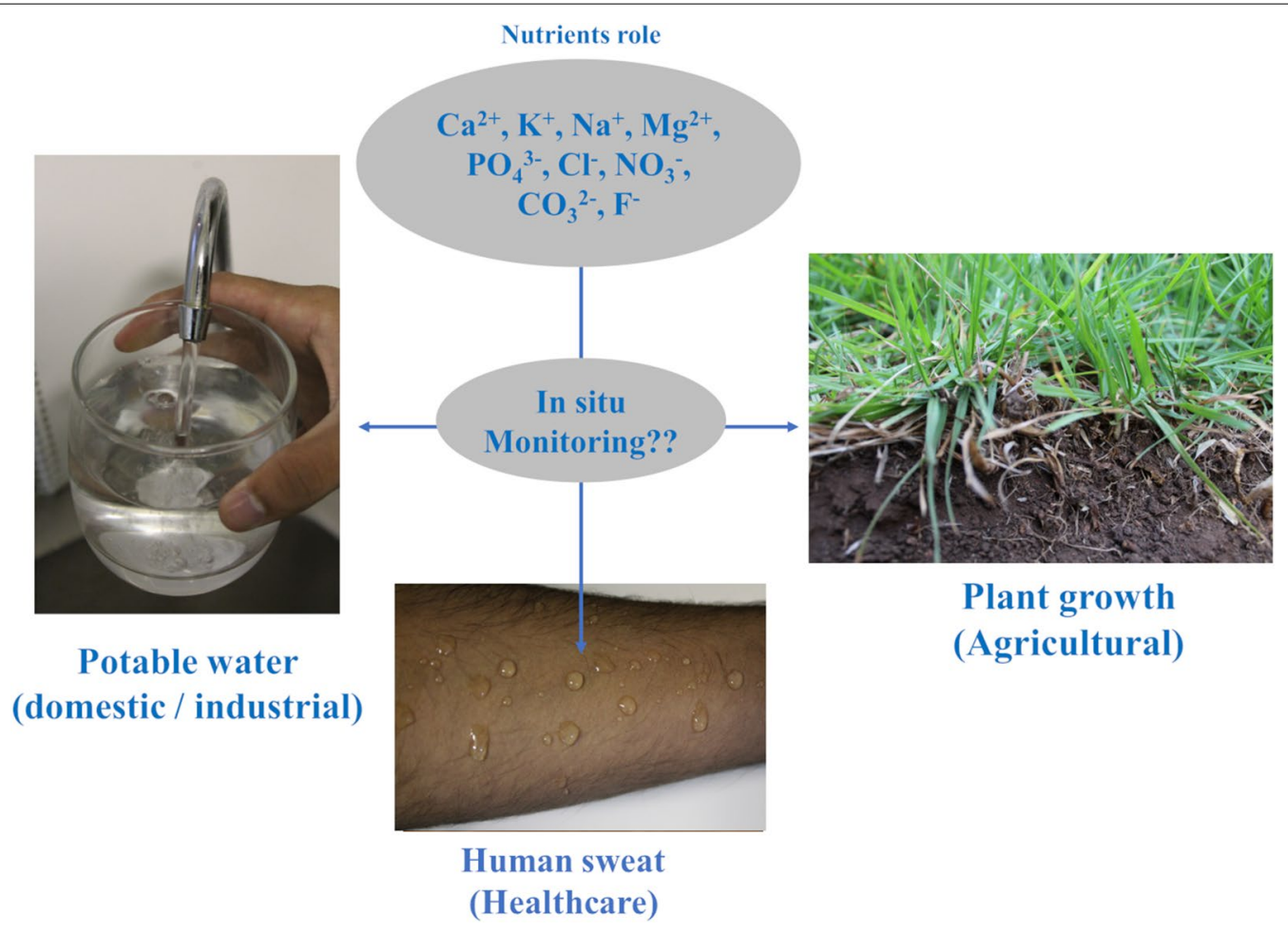

Fig. 1 Examples of where ions are found in systems that are important for society. lons (cations and anions) are present in aqueous solutions as potable water, soil water in agriculture, and human sweat (Images supplied by V. Sethumadhavan)

Table 1 Inherent properties and example applications of conducting polymers

\begin{tabular}{ll}
\hline An inherent property of conducting polymers with dopants & Applications \\
\hline Charge & Batteries or capacitor materials [2] \\
Optical absorption & Electrochromic displays [3] \\
Reactivity of electron-hole pairs & Organic light emitting diodes (OLED) [4] \\
Conductivity & Antistatic foils and electronic devices [5] \\
Structural change and volume & Actuators [6] \\
lonic conductivity & Membranes [7] \\
Biocompatibility & Tissue engineering applications [8] \\
Equilibrium potential & Corrosion protection [9] \\
Diffusion and adsorption & Compound release [10] \\
Work function, generation of electron-hole pairs & Components of organic solar cells [11] \\
Electrochemical reaction sites & Electrocatalysis [12] \\
\hline
\end{tabular}

Conducting polymers are generally defined by polymer chains that contain an extended conjugated backbone (alternating single and double bonded carbon atoms), with neighbouring chains aligned by $\pi-\pi$ stacking. The conjugated backbone defines an extended $\pi$-bonded structure, along which delocalized charges that can move. In the context of this review, the charges in the conjugated backbone are created/stabilized by insertion of ions near the polymer chain. In most cases, positive charges (holes) are stabilized in the $\pi$-bonded structure by doping the polymer with anions. This insertion process is known as doping, with the removal of anions known as dedoping; where doping accompanies the oxidation of the conducting polymer and dedoping leads to a reduction. It is this change in oxidation level that leads to the polymer properties being altered, hence providing a means by which to 'sense' the 
presence or absence of ions. A dedoped polymer may be redoped by exposing it to another reservoir of ions.

The properties of conducting polymers are dependent on the number of charge carriers within. These charge carriers depend on the number of ions doping the polymer. The influence of doping on the resultant properties is typically explained using the electronic bandgap model. In the fully reduced state, the conducting polymer will act as an insulator. Upon low levels of doping degenerate states are created within the energy gap, leading to a decrease in the bandgap and semiconducting behaviour being observed [13]. Some conducting polymers can display (semi-)metallic behaviour as the doping level increases towards the fully oxidized polymer and the bandgap disappears [14].

In parallel with the change in electronic properties, is the modification of the conducting polymers' optical properties [15-19]. While not the focus of this review, the changing optical absorption of conducting polymers with doping/dedoping, is exploited in electrochromic devices [20-25]. To connect these properties, it is often best to define the charged states within the polymer as neutral, polarons and bipolarons. The neutral segments of the conjugated backbone have, as the name suggests, no net charge. In the reduced form of the polymer these are the only species present. Zozoulenko et al. indicate that according to the convention in organic chemistry, polarons and bipolarons are usually referred to as radical cations and dications, respectively [26]. Therefore, the polarons are represented by a segment of the conjugated backbone containing a single positive chargebipolarons having two charges. Each of these charged states has a characteristic optical absorption. Given the relative amounts of each within the conducting polymer, which results from the level of doping, the overall optical absorption will be defined. The prototypical conducting polymer poly(3,4-ethylenedioxythiophene) is presented here as an example to link the electronic and optical properties (with suitability for sensing applications [27]). Traditionally it has been accepted that neutral, polaron and bipolaron segments in this conducting polymer have absorption peaks centred at approximately 600, 900 and $>1200 \mathrm{~nm}$ (Fig. 2a). While this approach has been widely used to describe the general behavior of the polymer, the recent work by Zozoulenko et al. has shed new light on this [26] (Fig. 2b). This recent research suggests that the optical properties, and the change with doping level, is far more complex than previously thought.

Both the electrical and optical change in conducting polymers can be used as transduction methods in sensing ions. This does not preclude other properties of the conducting polymer being monitored/measured, however few studies look at other properties of conducting polymers. One property that is studied (though not in the context of sensing) as a function of doping is the thermoelectric property [29-32].

The thermoelectric properties of a material relate to its ability to sustain/maintain an electrical potential that arises due to differences in temperature. It is this electrical potential that allows for current to flow to power an external electrical circuit. The parameter of interest here is the Seebeck coefficient (measured in V/K) [33]. Useful thermoelectric materials are those that have a high electrical conductivity and Seebeck coefficient, with a low thermal conductivity. Conducting polymers, such as poly(3,4-ethylenedioxythiophene), have been

\section{a Conventional (pre-DFT) interpretation}

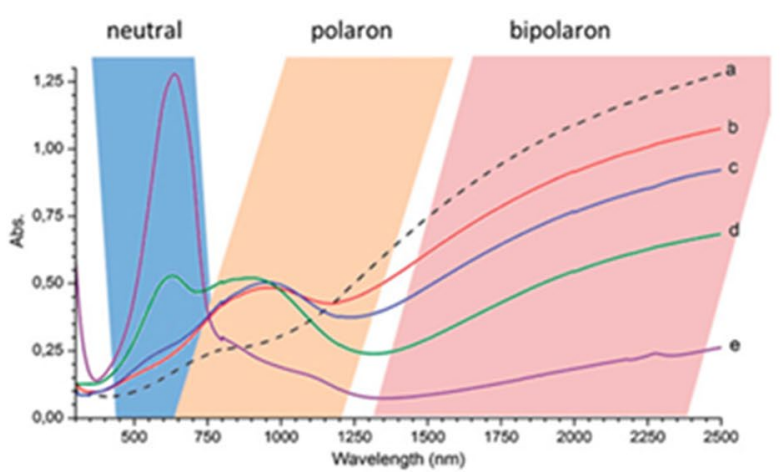

b New (DFT-based) interpretation

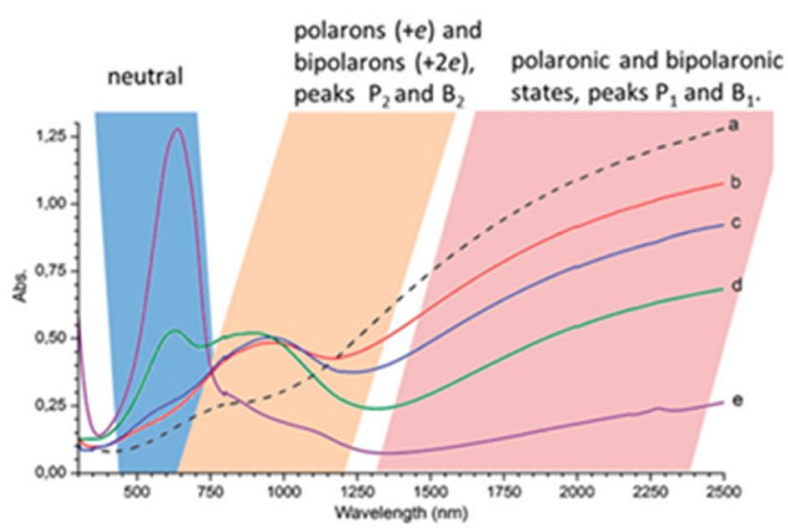

Fig. 2 Optical absorption spectra of the conducting polymer poly(3,4-ethylenedioxythiophene). Representative absorption spectrum of PEDOT ((Reproduced with permission from Ref. [28]. Copyright 2014 Royal Society of Chemistry), and its interpretation based on a the traditional pre-DFT picture and $\mathbf{b}$ our DFT calculations (Adapted with permission from reference from [26]. Copyright 2019 American Chemical Society)) 
explored for such applications [34]. In the context of this review, the Seebeck coefficient changes as a function of the doping level within the polymer (see $\alpha$ in Fig. 3). In the work of Bubnova et al. it has been demonstrated that for poly(3,4-ethylenedioxythiophene) doped with the tosylate anion the Seebeck coefficient changes over 1 order of magnitude [30]. Such a large change in this property as the doping level changes indicates the potential for its use in ion sensing.

Conducting polymers come in a range of different chemistries (see Table 2), beyond the aforementioned poly(3,4-ethylenedioxythiophene). Typically, all of these are polymerized from their starting monomer via an oxidative mechanism. Such a mechanism can be delivered through a range of techniques, from chemical to electrochemical to vapour processes. This review will not go into the details of each of these techniques (greater detail can be found in previous articles and reviews [35-40]). We would however like to emphasize that each of the different synthesis techniques lends themselves to the use of preferred doping anions during synthesis, as well as resulting in different structure/morphology. This will in turn impact on how the final doped conducting polymer behaves and performs in its interaction with ions (ultimately for sensing).

The conducting polymers are a subset of the broader class of conjugated polymers. It is worth mentioning that a range of conjugated polymers are also being researched for ion-sensing [41]. For example, Xu et al. have utilised electropolymerised conjugated polymers from aromatic molecules for sensing of different metals in the environment [42-45].

This review will focus on aspects of conducting polymers that define their use in ion sensing, with particular emphasis on literature from the past decade. Reviews covering details of the earlier work are contained in [4649]. Deliberately, the discussion will avoid gas sensing, as many gases are not charged (see other reviews [50-52]). To achieve a conducting polymer material that responds to ions, its physical and/or chemical structure needs to be modified, or controlled. The following section will generally discuss research into (physical and/or chemical) the structuring of conducting polymers.

\section{Structuring of conducting polymers}

Conducting polymers offer several possibilities for the development of ion sensors, however, they have some inherent limitations regarding solubility, long term stability and conductivity. To overcome these, conducting polymers have been modified, or hybridized with other heterogenous material components, which has led to materials with much improved properties and a range of new applications. When these modifications are successful, they lead to conducting polymers with high flexibilities, conductivities and mechanical stabilities, making these a key component in several applications, including sensors [53]. Various approaches have been taken in the synthesis of such hybrid materials and of particular significance is the template used in the synthesis reaction,

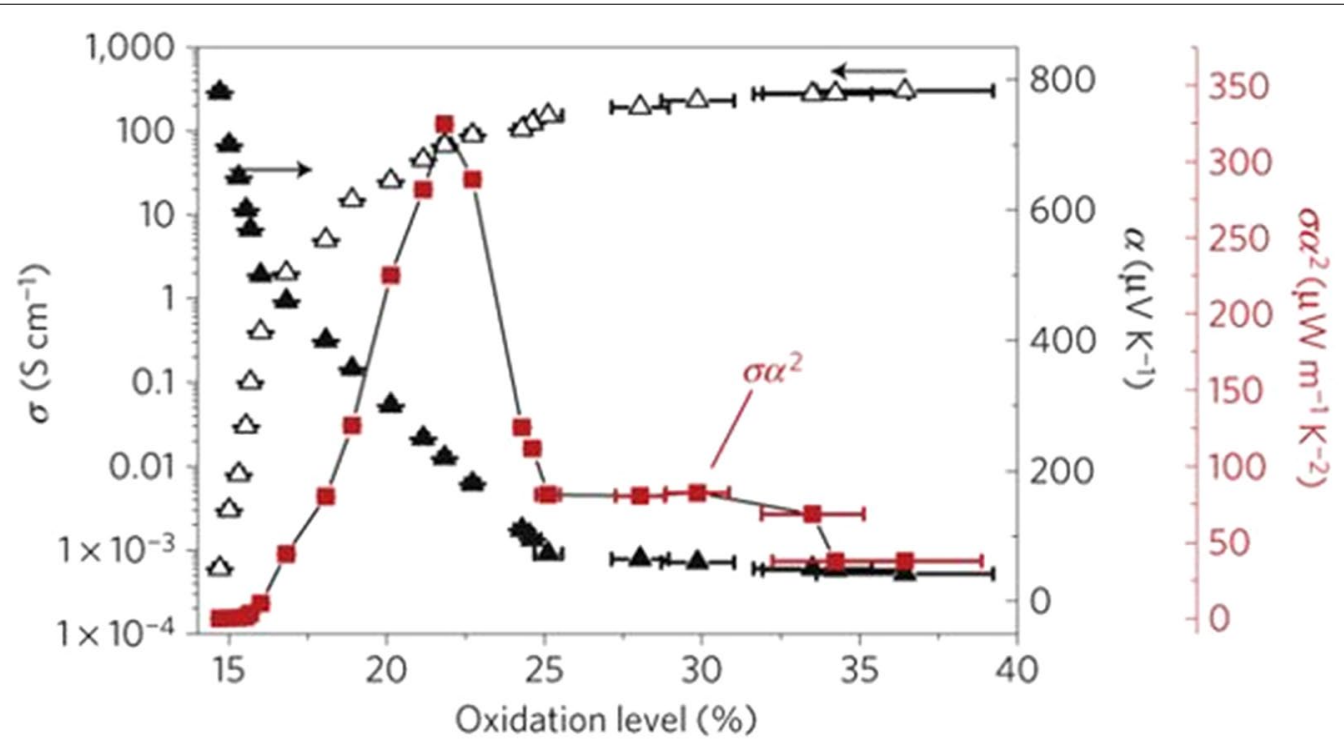

Fig. 3 Thermoelectric properties of poly(3,4-ethylenedioxythiophene) with oxidation level. The change in Seebeck coefficient a (filled triangles), electrical conductivity $\sigma$ (open triangles) and corresponding power factor $\sigma a^{2}$ (red squares) of the conducting polymer poly(3,4-ethylenedioxy thiophene) as a function of its oxidation (doping) level (Reproduced with permission from [30]. Copyright 2011 Springer Nature) 
Table 2 Structures and names of several commonly studied and readily available conducting polymers

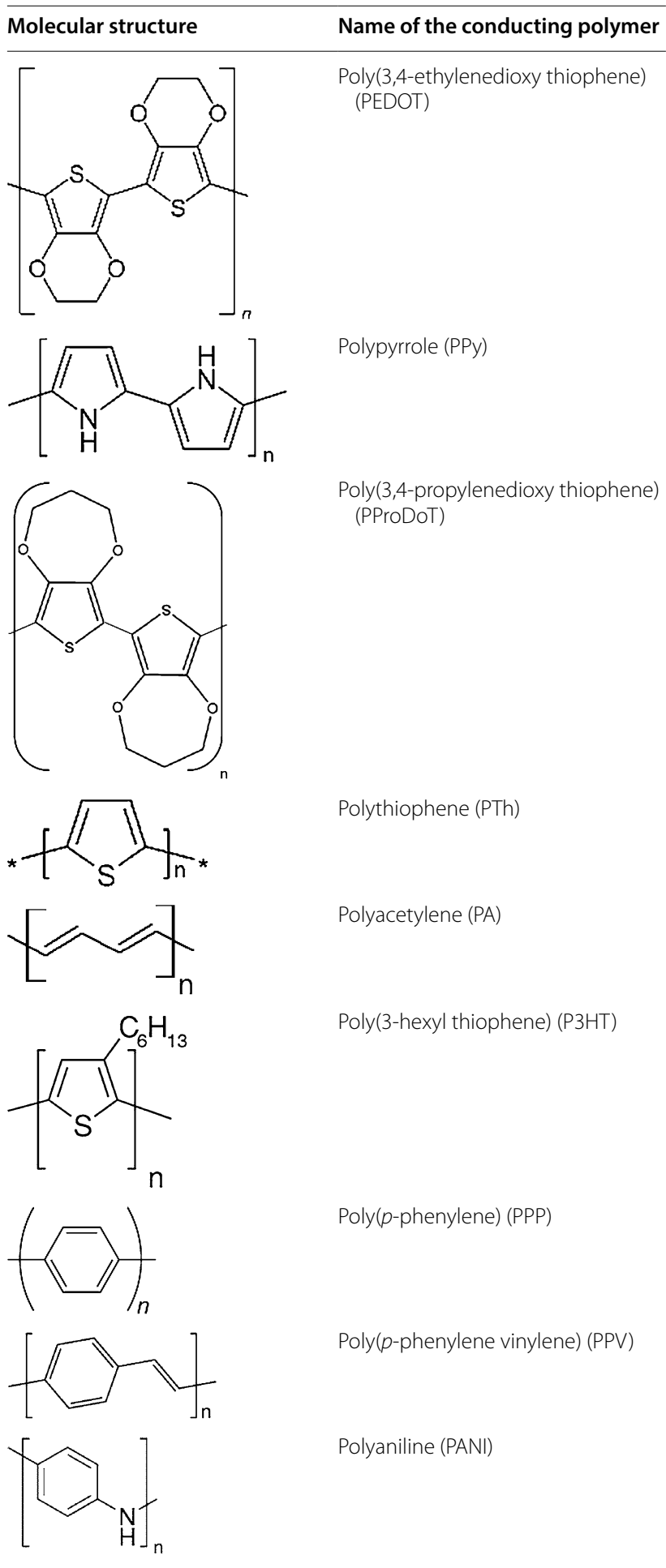

All the polymers contain a conjugated backbone of alternating single and double bonded carbon and/or heteroatoms the oxidizing agent, and the order of addition of each component.

Looking specifically at conducting polymer applications in chemical and biochemical sensors, there have been a variety of approaches to their modifications dating back to the mid-1980s. One of the more useful approaches to improve the selectivity of resulting chemical sensors, is via the functionalization of the conducting polymers. Polymerization of dibenzo-crown ethers with cation-complexing properties opened a new way for the construction of ion-complexing conducting polymers [54]. By using functional dopants, such as tetrasulfonated dibenzo-18-crown-6 [55], or by covalent binding of ion recognition sites as side-groups to the conducting polymer backbone [56, 57], ion-selectivity was introduced into conducting polymers. Other types of chemical sensors have been possible by using other functional groups attached to conducting polymers.

Since oxidized conductive polymers have a polycationic backbone, they can therefore serve as anion exchangers, which results in an anionic potential response. We know that various small anions can contribute to the measured potential (such as chloride, bromide, nitrate, perchlorate and thiocyanate), which implies that the anionic response of conductive polymers is non-selective [58, 59]. However, some conducting polymers may possess selectivity for specific ions, such as a proton $\left(\mathrm{H}^{+}\right)$and perchlorate $[60,61]$.

Immobilization of doping anions to obtain an excess negative charge gives a conducting polymer with cationexchange behaviour, which results in a cationic potentiometric response [62]. The potentiometric response originates partly from the chemical structure of the conducting polymer backbone and partly from the inserted doping ions. In order to enhance the selectivity to the target analyte, it is possible to modify the chemical structure of the conducting polymer by covalent bonding of suitable receptors, or by the immobilization of functional dopants that are selective to the target analyte.

Dopant ions are generally introduced into the polymer system during chemical or electrochemical polymerization. They play an important role in balancing the charge distribution within the polymer, and doped polymers show increased electrical conductance. The resultant morphology of the material is reflected in the type of dopant ion used. An example showing the difference in surface morphology between polypyrrole films doped with tetrafluoroborate with those doped with tosylate are shown in Fig. 4 [63].

Data from the literature confirms a change of dopant within the conducting polymer has a large effect on the sensor sensitivity by changing a physical transduction property. Conversely the different dopant ion does not 

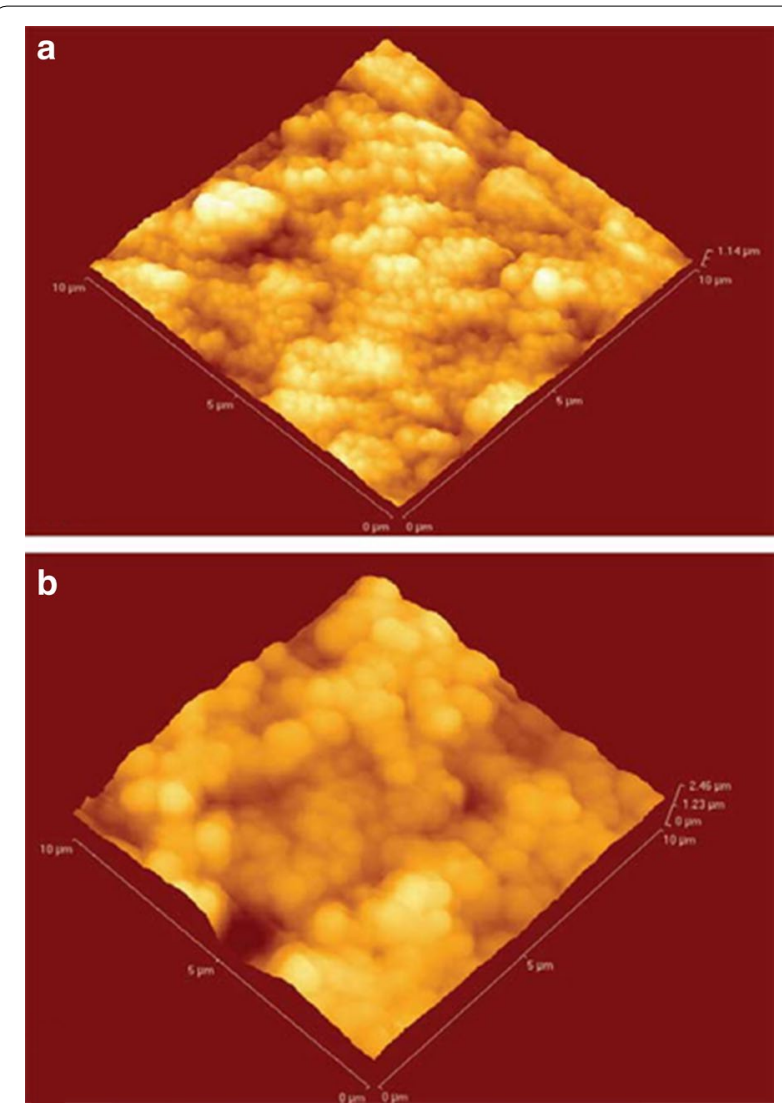

Fig. 4 Changing polymer morphology with different doping ions. Comparison of atomic force microscopy (AFM) images of the surface morphology of polypyrrole doped with different ions: a polypyrrole tetrafluoroborate; and b polypyrrole $p$-toluenesulfonate (Reproduced with permission from [63]. CC BY-NC-ND 3.0)

markedly affect the ion selectivity of the polymer. In order to achieve new selectivity by changing the dopant ion, some researchers have explored functionalised dopants (see within this review for examples [47]). These dopants then directly interact with the ion of interest, while the properties of the conducting polymer are still used to quantify the ion concentration.

Another method of polymer modification is via postpolymerization functionalization. Today, the most widely used tool for post polymerization functionalization is 'click' chemistry. Wei et al. [64] have carried out post polymerization functionalization using thiolene click chemistry, on poly(3,4-propylenedioxythiophene) (PProDOT). Modification of $\mathrm{P}$ (ProDOT-diene) with polyethylene oxide thiol (o-(2-mercaptoethyl)-o'-methylhexa(ethylene glycol)) showed (via cyclic voltammetry) that both the redox peak and oxidation onset potentials in the modified polymer film had shifted to a more positive potential. The ion transport enabled by the extra chain length of thiol, also leads to a much larger charge

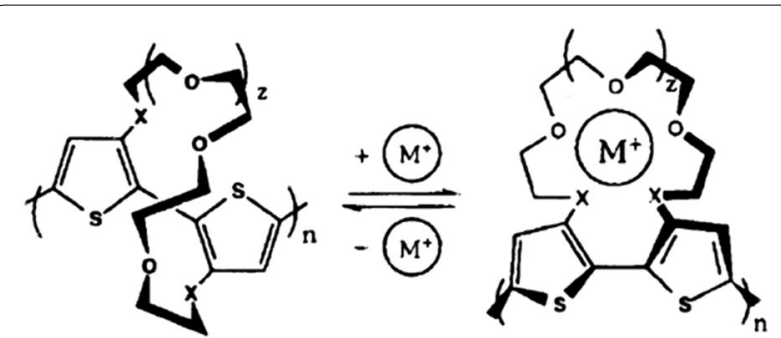

Fig. 5 Modified chemical structure leading to physical changes upon ion insertion. Schematic of polymer backbone conformational changes induced by metal ion complexation (Reproduced with permission from [67]. Copyright 1993 American Chemical Society)

storage capacity of the polymer film. Overall this method facilitates fine-tuning of the surface chemistry of these films, leading to improved charge transfer and wettability, as well as enhanced ion selectivity.

The term charge transfer refers to a succession of interactions between two molecules, ranging from a very weak donor-acceptor dipolar interactions to interactions that result in the formation of an ion pair, depending on the extent of electron delocalization. Charge transfer complexes are formed between electron-rich donor molecules and electron deficient acceptors. Typically, donor molecules are electron rich heterocycles [65] (e.g., furan, pyrrole, thiophene), aromatics with electron-donating substituents, or compounds with free, nonbonding pairs of electrons. Acceptor molecules are electron deficient systems such as purines and pyrimidines or aromatics with electron-withdrawing substituents.

Conducting polymers can be functionalized with crown ethers for the selectivity of cations using an electrochemical technique. In 1989, Roncali and co-workers synthesized poly[3-(3,6-dioxyheptyl) thiophene and evaluated the electrochemical properties in the presence of tetra- $n$-butylammonium and lithium electrolytes [66]. The author suggested that the conformal changes occurred in the polymer backbone due to the exchange of electrolytes and the effect of solvent, which influences the ion selectivity during the process. Swager et al. developed a conducting polymer with a crown ether receptor covalently attached to the thiophene complex, as shown in Fig. 5, for the sensing of metal cations (lithium, potassium, sodium) with respect to ion size [67]. The diameter of the crown ether cavity relates to the ionic size of the metal cation and the oxygen atom retards in electrostatic interaction with the metal cation, due to its electronegativity and lone pair electrons. The same research group also functionalized a crown ether with a bithiophene complex for the selectivity of lithium ions and found it to result in conformal changes and lower conductivity [68]. 
Incorporation of pyridyl based ligands in conducting polymers showed better functionality for the development of sensors [69-71]. These ligands are placed in direct- $\pi$ communication with the backbone tethered by alkyl chains [72]. Coordination of metal ions may lead to an alteration in the conformal change and it affects the electrical, optical, and electrochemical properties of conducting polymers. In this project, functional complexes were incorporated in the conducting polymers and were used to define the optical properties and structural morphology of conducting polymers with respect to ion uptake.

Such approaches to manipulate the polymer's structure can be exploited to improve the material's function for a particular application and characterized to improve our understanding of the material's properties. The following section will explore ion interactions with conducting polymers in more detail.

\section{lon interactions with conducting polymers}

The mechanism and behaviour of the oxidation and reduction in conducting polymers, has an influence on the polymer's utility in sensing. To this end, research into the doping process is of interest that accompanies the oxidation or reduction of the polymer. This section will discuss this research, though not necessarily restricting it to systems that result in sensing.

\section{Polymer properties defined by doping ions}

As indicated in the Introduction, the properties of a conducting polymer depend on the doping level of ions within. The type of ion used to dope the conducting polymer has been reported to impact on the resultant properties as well.

In the late 1980's, Warren et al. [73] electropolymerized polypyrrole (and poly-3-methylthiophene) from an extensive range of electrolyte solutions. By changing the electrolyte in which the conducting polymers were synthesized, a significant difference in electrical conductivity was observed (3 orders of magnitude for polypyrrole). By creating free-standing thin films for X-ray diffraction and transmission electron microscopy (TEM) analysis, the resultant change in structure and morphology was rationalised as the reason for the property change. In this case, it was concluded that the dopant anions define different structures within the conducting polymer, and thus result in different electrical properties.

To highlight the complexity of structure-property relationships in doped conducting polymers, slightly contrary conclusions were drawn by Hao et al. for doped polyaniline [74]. Herein a range of anions (camphorsulphonate, tosylate, chloride, perchlorate, sulphate and phosphate) were exchanged within the polymer thin film using electrochemical cycling. While the exchange was observed via the changes in the electrochemical and optical properties of the polymer, morphology studies indicated that the property changes were independent of any changes in structure. This may originate from differences in the inherent polymer behavior owing to the different chemistry between polyaniline and polypyrrole.

Further to this discussion, Rudd et al. used a vapor polymerization process to fabricate and study poly(3,4ethylenedioxythiophene) [75]. They showed, both theoretically and experimentally, the influence of different dopants on the properties of poly(3,4-ethylenedioxythiophene). By using an electrochemical redox process, they removed tosylate ions used during polymerization and exchanged them with other anions of interest. When tosylate (redoping), perchlorate and nitrate are employed, they achieve the same doping level yet differing electrical and optical properties. Similarly, despite a lower doping level, chloride and phenylphosphate also resulted in differing properties. Density functional theory calculations indicated that the electron density along the polymer chain is similar for each inserted anion. X-ray diffractograms suggested that the anions impart subtly different chain stacking/packing, that in turn define different conducting polymer properties (supporting the statements of Warren et al. for polypyrrole).

Given the apparent link between the polymer structure and the resultant properties, Rolland et al. [76] undertook a theoretical study to link the morphology and charge carrier mobility for poly(3,4-ethylenedioxythiophene) doped with tosylate. Central to this study was determining whether crystallinity was a sufficient enough criterion for good charge transport within the conducting polymer. The study concluded that $\pi-\pi$ stacking of the polymer chains was the most important factor in defining the charge transport properties, rather than a highly crystalline structure. In fact, high levels of $\pi-\pi$ connections between chains that lead to a percolated network facilitated high charge carrier mobility (hence high electrical conductivity). It could be inferred that those doping ions that enhance the $\pi-\pi$ interactions between neighbouring polymer chains, will be most successful at enhancing the electrical conductivity (or similar properties).

It is possible to exchange the doping ions within the conducting polymer through chemical treatment, rather than electrochemically. Inganas et al. [77], showed the (reversible) ion exchange mechanism in polypyrrole, by treating it with an alkaline solution. They showed that the alkaline treatment decreases the electrical conductivity and changes the optical properties. It is rationalized that deprotonation/protonation of the nitrogen in the pyrrole unit, defines whether a single electron remains on the conjugated backbone (allowing for recombination 
with holes). Crispin et al. [78] undertook a similar study using vapor deposited poly(3,4-ethylenedioxythiophene) doped with tosylate. Ion exchange was achieved by exposure to hydrochloric acid or sodium hydroxide solutions. The chloride or hydroxide ions imparted changes in the packing structure of the conducting polymer. However, this alone could not explain the changes in the polymers' thermoelectric properties. Beyond the changes in structure, the oxidation level of the polymer also changed. This highlights that the anions themselves play a critical role in defining the conducting polymer properties, through modification of the oxidation level and the structure/ morphology. More specifically the conducting polymers have a primary redox state that relates to electron transfer and many secondary states or transitions that relate to other changes in molecular structure. In a similar manner, de Geyer and co-workers utilised sulfuric acid treatment to dramatically enhance the electrical conductivity of poly(3,4-ethylenedioxythiophene) doped with triflate anions [79]. The hydrogen sulfate ions have less steric hinderance than triflate, increasing the oxidation level and the structural order.

From the perspective of future research, the interplay between ion properties, doping level, oxidation level, and structure, will be critical to understanding how doped conducting polymers behave. From this understanding should come utility in ion sensing with conducting polymers. Thus far the discussion has focused on the equilibrium state of the conducting polymer at the respective doping level of interest. However, in the context of sensing it is also important to understand the dynamics of the doping process.

\section{Dynamics of doping/dedoping process}

In the process of sensing, the active material will generally be utilised in some finite volume-be it a thin film, all the way through to a bulk volume of material. Thus, the ability for ions to easily and readily diffuse in and out of the material becomes an important consideration. This section will discuss recent work exploring the doping/ dedoping process in conducting polymers.

Early work of Kaufman, Kanazawa and Street, presented a gravimetric electrochemical voltage spectroscopy technique to study the process of doping and dedoping of polypyrrole in a lithium perchlorate electrolyte [80]. For this system they show that the mass of the polymer film increases upon full electrochemical reduction. The increase in mass leads to the conclusion that cations (lithium) are taken up by the material, leading to charge neutralisation of the anions through the creation of a salt. In the process, prior to full reduction, some perchlorate liberation is observed, which transitions into lithium uptake at greater levels of reduction. This indicates that only a proportion of the perchlorate ions are mobile and hence able to diffuse from the polymer structure. The remaining perchlorate act as sites for the highly mobile lithium to bind. The reverse is true upon oxidation, in that lithium is initially expelled prior to the uptake of perchlorate at higher oxidation levels. When tosylate is used as the anion (with lithium as the cation) in the same experiments, there is mass loss at all stages of the electrochemical reduction process. This indicates the tosylate is expelled from the polypyrrole at all reduction levels, and no cation uptake is observed. Pel and Inganas, in a later study using volume changes observed via bending of a biopolymer strip, substantiate the mass transport and insertion of cations during the reduction process [81]. More recently, Latonen et al. [82] explored the ion exchange behaviour of polypyrrole doped with large anions. In this case the anion is immobile within the conducting polymer matrix-leading to anion-cation interaction driving the uptake of cations upon electrochemical reduction.

In the case of poly(3,4-ethylenedioxythiophene) doped with polystyrene sulfonate, the polyanion is also relatively immobile-allowing for ionic mobility of cations within. Stavrinidou et al. explored the mobility of various cations within thin films of as-deposited and crosslinked polymers [83]. They show that a variety of cations (protons, potassium, sodium and choline) have increased mobility within the conducting polymer matrix compared to that in bulk water (highlighting electro-osmotic contributions). Also, as the hydration sphere of the cation increases, its mobility within the conducting polymer matrix decreases. Adding to this complexity of anion vs. cation diffusion into or out of the conducting polymer, evidence also exists that counter flows of solvent are also present [84]. Hence, the relative diffusion rates of ions and solvent in and out of the conducting polymer are important for the doping/dedoping process.

Modarresi et al. have theoretically investigated the diffusion coefficient and the position of ions within poly(3,4-ethylenedioxythiophene) doped with tosylate [85]. Their study reports the diffusion of both cations (sodium) and anions (chloride) in and around the polymer matrix, based on the overall hydration level of the system (Fig. 6). This takes into account the complex interaction between the conducting polymer and the ions, as well as the contribution of the solvent. They demonstrated that high levels of hydration, e.g. $80 \%$, lead to diffusion of ions away from the polymer matrix. However, a low level of hydration confines the ions in the vicinity of the polymer matrix and consequently, lowers diffusion coefficients. It has been hypothesized that the evaporation of water leads to a decrease in the crystallite size, and simultaneously causes the higher oxidation level. 

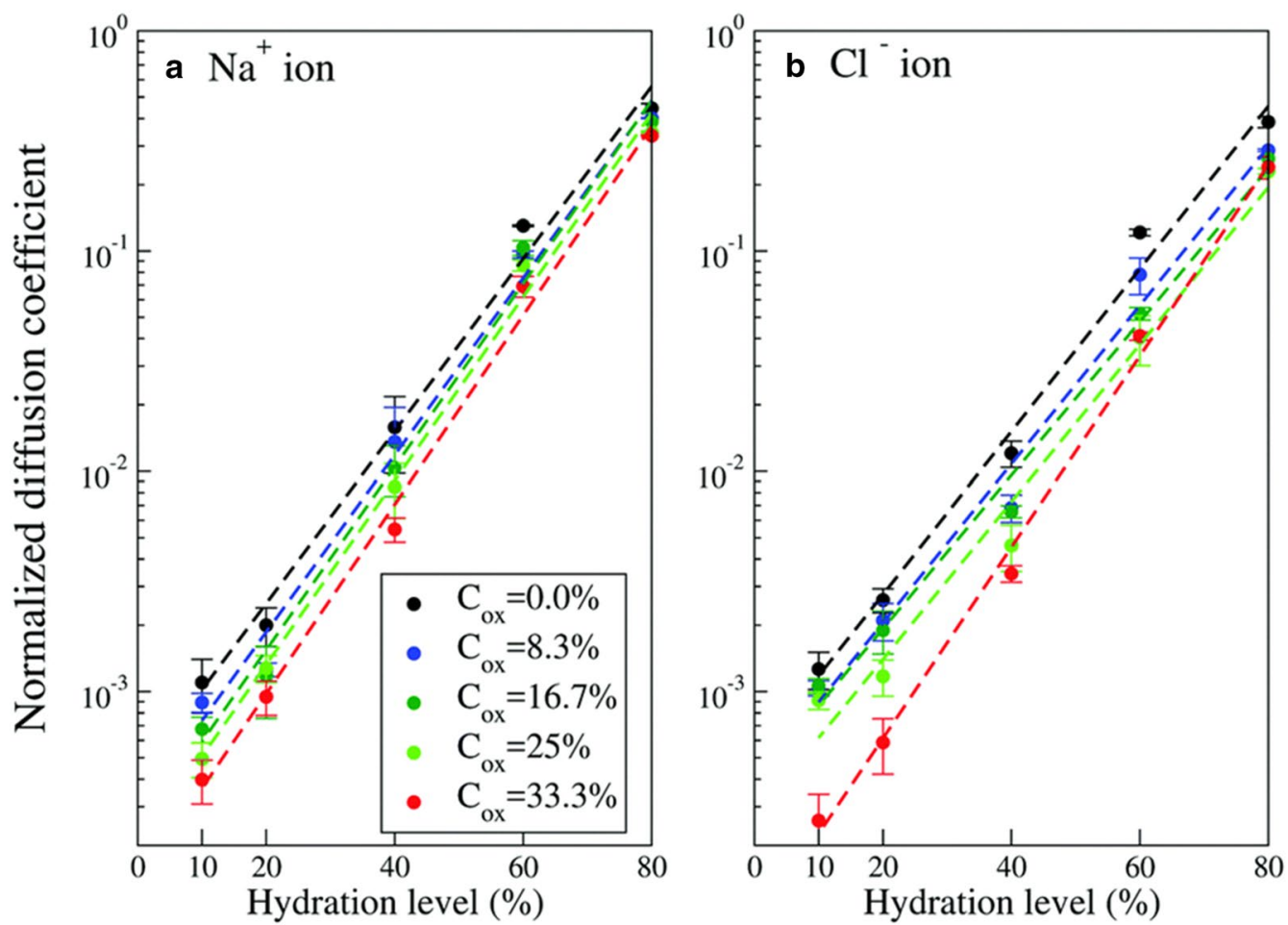

Fig. 6 Diffusion of ions in a conducting polymer. Calculated diffusion coefficients for a sodium and $\mathbf{b}$ chloride in tosylate doped poly 3,4-ethylenedioxythiophene (Reproduced with permission from [85]. CC BY 3.0.)

Given that many sensing applications will be at high hydration levels (aqueous systems), the ionic diffusion at high hydration is of interest. As highlighted in Fig. 6, at a given hydration level the cations are only slightly more mobile within the conducting polymer. Similarly, as the doping level of the polymer increases (shown as increasing $\mathrm{C}_{\mathrm{ox}}$ ), the diffusion coefficient decreases-relatively greater decreases for chloride compared to sodium.

This observation is predicted from analysis of the modified Nernst-Planck equation [86]. In the case of the diffusion-migration model for coupled transport (both electronic and ionic charge carriers), the enhancement of inter-site hopping (of electronic charge carriers) increases as the mobility of counter ions decreases. As the doping level increases then migration (ion diffusion) diminishes. With a better understanding of how ions can interact with conducting polymers and the techniques by which to control their structure, it is possible to use these to sense and differentiate between ions.

\section{lon sensing in conducting polymers}

Ion sensors perform three functions; (i) analyte (molecular) recognition [87], (ii) transduction and (iii) signalling. These functions are performed in parallel, where the analyte recognition induces a measurable change in the properties of materials (transduction) that can be quantified using some form of (opto-) electronic signalling system.

Molecular recognition: Analyte interacts with the receptor material and shows affinity for the selective analyte under chemical reaction. A recognition element (R) can be designed, in which an analyte (A) and a receptor interacts and gives the product of analyte-receptor interaction (P) under chemical equilibrium [87],

$$
\mathrm{A}(\text { Sample })+\mathrm{R}(\text { Recognition element }) \rightleftharpoons \mathrm{P} \text { (Interaction) }
$$

Transduction: Involves the transfer of chemical information to measurable electrical (change in electrical signal), optical (change in colour) or thermal (change in heat) signals, which in turn define the quantity of absorbed specific analyte [88].

Signalling: Involves the signal processing analysis of transduction information to digital/analog (e.g. capacitance/resistance) output.

A common ion sensing approach is use of ion selective electrodes (ISE). Typically, ISEs are membrane electrodes that produce an electrical potential by converting the activity of ions dissolved in a solution. The change in potential can be measured with the help of an electrical circuit (such as a voltmeter, or potentiostat) and reflects 
the change in selective ion concentration [89]. In 1906, Cremer discovered the first ISE by using a glass electrode to measure the $\mathrm{pH}$ of an aqueous solution [90].

Ion selective materials form the central component in ISEs, which in turn are central to the overall functionality of the ion sensors themselves. A common class of ion selective materials are known as ionophores. Despite there being many known ionophores showing specific anion interactions [91-99], there are some limitations to their use in practical devices. These limitations are listed below [100];

- Ion selective materials can be fouled by chemical or biological species and other organic solutes.

- Interference by other ions.

- Electrodes may be fragile and have limited shelf life.

In an attempt to overcome these limitations, conducting polymers can be used as the ion selective material and also as a transducer, because they are environmentally stable and electrically conductive. Conducting polymers show strong promise to replace metal-based materials that can be easily corroded by the surrounding environment. Much work is still required however to assess the issues of interference and fouling. Furthermore, it may be beneficial to combine the ionophores into or onto the conducting polymers to realise efficiency and/or stability gains in the overall device.

Bobacka et al. have extensively studied ion sensing and conducting polymers. For example, they describe the selectivity of silver ions with poly-3-octyl thiophene [101]. While the observation that immobile and lipophilic anions within the conducting polymer matrix showing sub-Nernstian responses to silver is logical, the potentiometric response of undoped polymer to silver is less so. This implies an inherent property of the conducting polymer to directly interact with the cation, as opposed to the electrostatic anion-cation interaction being the primary mechanism. In this case, silver is known to interact with sulphur (heteroatom in the thiophene) and carbon-carbon double bonds ( $\pi$-bonded structure of the polymer). Exploiting this type of interaction between cations and conducting polymers, they employed poly(3,4-ethylenedioxythiophene) as an ion-to-electron transducer in a calcium-selective electrode [102]. In relation to the anions, they very recently showed that polypyrrole membranes (doped with tosylate, camphorsulphonate, or hexafluorophosphate) could not only be used to control anion transport, but to separate monovalent from divalent anions [103]. As a practical example, toxic hydrogen arsenate could be separated from nitrate and chloride using electrochemical switching. Studies such as this give insight into a range of yet unexplored behaviours, mechanisms and applications for conducting polymers interacting with ions.

Bomar et al. developed a nitrate selective electrode by using electropolymerized $\mathrm{N}$-methyl pyrrole in a supporting electrolyte of potassium nitrate [104]. The reported advantage of using $\mathrm{N}$-methyl pyrrole, over pyrrole alone, is that the attached methyl group should hinder protonation of the nitrogen atom in acidic media. Choosing electropolymerization to prepare the conducting polymer resulted in a high degree of branching and cross linking that lead to mechanical stability under electrochemical cycling. The authors refer to this as an ion-imprinted polymer which yields a superior ISE over more traditional nitrate selective variants. Hyodo et al. investigated the same $\mathrm{N}$-methyl pyrrole for sulphate ion sensing [105]. Unfortunately, over-oxidation of the polymer was observed, with the formation of carbonyl groups together with associated structural change. This highlights that tailoring of the conducting polymer, doping anion, and resultant structure, are all key to specific ion sensing.

With respect to nitrate sensing, Rudd et al. [106] fabricated poly(3,4-ethylenedioxythiophene) doped with tosylate using a vapor deposition process, which was found to selectively uptake nitrate ions in the aqueous environment. The novelty of the work prescribed that, when these films start in the electrochemically reduced state, nitrate ions are selectively uptaken in the polymer matrix without any electrical methods to oxidise the poly(3,4-ethylenedioxythiophene). It also showed an in situ monitoring mechanism for real time sensing applications. An experimental analysis of anion concentration from a water sample taken from agricultural land is shown in Fig. 7. The results from an X-ray photoelectron spectroscopy (XPS) analysis, demonstrated that only nitrate was present (no other analytes).

Upon further analysis, it was found that the concentration of nitrate increases with the increase in conductivity of reduced poly(3,4-ethylenedioxythiophene), and was later confirmed using ion chromatography. From the analysis, the authors hypothesized that $\pi-\pi$ interaction occurs between the anions and the aromatic ring of poly(3,4-ethylenedioxythiophene) and deals with supramolecular chemistry.

In more recent studies Rudd et al. reported the structure-property-performance of vapor phase polymerized poly(3,4-ethylenedioxythiophene) for sensing nitrate ions [107]. In order to understand this mechanism, poly $(3,4-$ ethylenedioxythiophene) samples were prepared by changing several aspects of the oxidant solution (concentration of oxidant, solvent used, and additives employed). They hypothesized that the change in the polymerization kinetics leads to a change in the morphology, structure and electrical conductivity. Among the electrical 


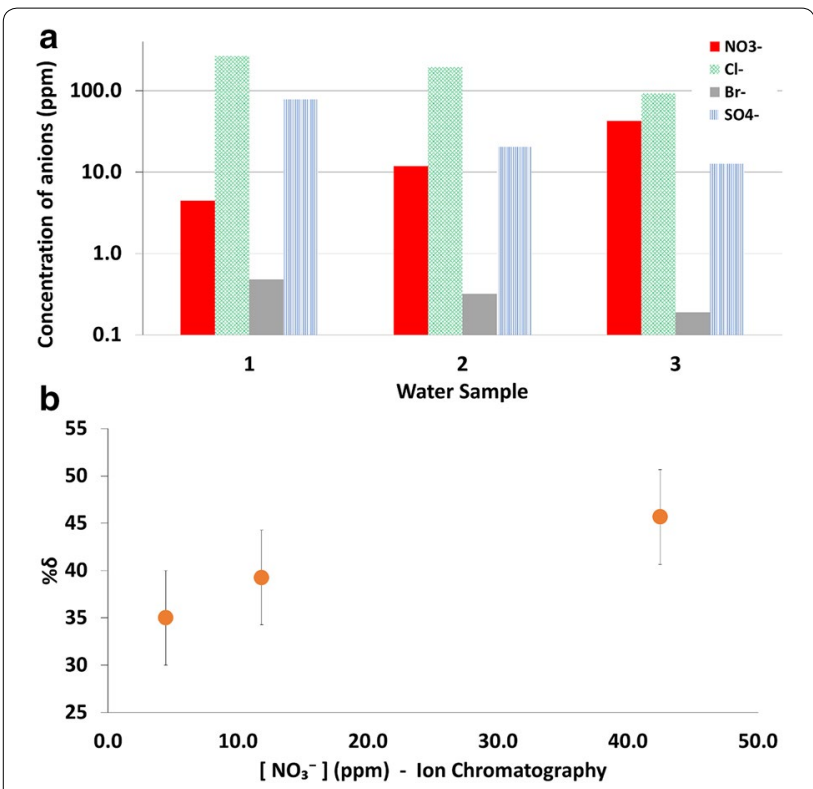

Fig. 7 Sensing nitrate in ground water. a Concentration of anions present in the water sample from agricultural area. $\mathbf{b}$ Concentration of nitrate ion vs. poly(3,4-ethylenedioxythiophene) sheet resistance (Reproduced with permission from [106]. CC BY 4.0)

properties of vapor phase polymerized poly(3,4-ethylenedioxythiophene), the structure and morphology may also play a role in the selectivity for nitrate. Further analysis with X-ray diffraction indicated that an increase in the ordering of poly(3,4-ethylenedioxythiophene) is proportional to the doping level of anions.

Hence, the structure-property relationships of other conducting polymers need to be investigated for further development of conducting polymer based ion sensors.

\section{Conclusion}

Conducting polymers are interesting materials with properties that depend on their doping level and structure. Doping being the insertion of ions into the polymer structure, which in turn affects the oxidation level of the conducting polymer. Because the conducting polymer properties change as a function of their oxidation, they show promise for ion sensing.

Researchers have shown that a general set of rules for how conducting polymers and ions interact is difficult to define. That is, several factors influence the interaction. These range from the chemistry of the conducting polymer to the method of fabrication. The choice of the counterion used during fabrication is just as critical as the specific ion that is chosen to be sensed.

The interaction itself is also not easily defined, as large anions within the conducting polymer matrix are relatively immobile, and hence lead to the material interacting with cations within the neighboring solution. Conversely, smaller anions spontaneously exchange with the electrolyte inferring anion sensing with the conducting polymer. In parallel with ion movement in and out of the polymer, there is also evidence to suggest solvent flux also.

When the functionality and nature of the anion or conducting polymer are modified to include other interaction mechanisms (beyond Coulombic attraction), then further sensing opportunities arise. These include $\pi$-ion interactions between the ion of interest and the conjugated backbone of the conducting polymer.

As the fundamental understanding of these materials is expanded, along with the resultant properties, which can be modified and measured (optical, electrical, optoelectronic, electrochemical, thermoelectric, etc.), the ways in which ion sensing can be achieved with conducting polymers will also suitably expand.

\section{Abbreviations}

AFM: atomic force microscopy; ISE: ion selective electrode; P(ProDOT): poly(3,4-propylenedioxythiophene); TEM: transmission electron microscopy; XPS: X-ray photoelectron spectroscopy.

\section{Acknowledgements}

Not applicable.

\section{Authors' contributions}

VS, SR, ES, KZ, PT and DE jointly wrote the manuscript. All authors read and approved the final manuscript.

\section{Funding}

SR acknowledges the support of the Commonwealth Government of Australia through the Innovation Connections Grant (ICG000582). DE acknowledges the support of the Australian Research Council (DP170104367, FT160100300). The funding body did not play any role in the design of the study or collection, analysis, or interpretation of data, nor in the writing the manuscript.

\section{Availability of data and materials} Not applicable.

\section{Competing interests}

The authors declare that they have no competing interests.

\section{Author details}

1 Future Industries Institute, University of South Australia, Mawson Lakes, SA 5095, Australia. ${ }^{2}$ School of Natural and Built Environments, University of South Australia, Mawson Lakes, SA 5095, Australia.

Received: 6 May 2019 Accepted: 24 October 2019

Published online: 21 November 2019

References

1. Antonijevic MM. Ion sensors. Sensors. 2002;1(2):383.

2. Naegele D, Bittihn R. Electrically conductive polymers as rechargeable battery electrodes. Solid State lonics. 1988;28(2):983-9.

3. Wen LA, Baohua G, Mattes QR. Fabricating conducting polymer electrochromic devices using ionic liquids. J Electrochem Soc. 2004;151(2):33-9.

4. Alsalhi MS, Alam J, Dass LA, Raja M. Recent advances in conjugated polymers for light emitting devices. Int J Mol Sci. 2011;12(3):2036-54. 
5. Song E, Choi JW. Conducting polyaniline nanowire and its applications in chemiresistive sensing. Nanomaterials. 2013;3(3):498-523.

6. Kaneto K, Fujisue H, Kunifusa M, Takashima W. Conducting polymer soft actuators based on polypyrrole films-energy conversion efficiency. Smart Mater Struct. 2007;16(2):S250-5.

7. Pellegrino J. The use of conducting polymers in membrane-based separations. Ann N Y Acad Sci. 2003;984:289-305.

8. Guo B, Ma PX. Conducting polymers for tissue engineering. Biomacromol. 2018:19(6):1764-82.

9. Ohtsuka T. Corrosion protection of steels by conducting polymer coating. Int J Corros. 2012;2012:1-7.

10. Lira LM, de Córdoba Torresi SI. Conducting polymer-hydrogel composites for electrochemical release devices: synthesis and characterization of semi-interpenetrating polyaniline-polyacrylamide networks. Electrochem Commun. 2005;7(7):717-23.

11. Tadesse T. Application of conjugated organic polymers for photovoltaic's: review. J Phys Chem Biophys. 2018. https://doi.org/10.4172/21610398.1000263.

12. Albertas M. Electrocatalysis at conducting polymers. Synth Met. 1999:107:75-83.

13. Jaiswal M, Menon R. Polymer electronic materials: a review of charge transport. Polym Int. 2006;55(12):1371-84.

14. Bubnova O, Khan ZU, Wang H, Braun S, Evans DR, Fabretto M, et al. Semi-metallic polymers. Nat Mater. 2014;13(2):190.

15. Håkansson E, Lin T, Wang H, Kaynak A. The effects of dye dopants on the conductivity and optical absorption properties of polypyrrole. Synth Met. 2006:156(18-20):1194-202.

16. Kaneto $K$, Yoshino $K$, Inuishi Y. Electrical and optical properties of polythiophene prepared by electrochemical polymerization. Solid State Commun. 1983;46(5):389-91.

17. Bredas J, Scott J, Yakushi K, Street G. Polarons and bipolarons in polypyrrole: evolution of the band structure and optical spectrum upon doing. Phys Rev B. 1984;30(2):1023.

18. Volkov AV, Singh SK, Stavrinidou E, Gabrielsson R, Franco-Gonzalez JF, Cruce A, et al. Spectroelectrochemistry and nature of charge carriers in self-doped conducting polymer. Adv Electron Mater. 2017;3(8):1700096.

19. Xia Y, Ouyang J. Transparent conducting polymers. Organic optoelectronic materials. Berlin: Springer; 2015. p. 359-92.

20. Panero S, Passerini S, Scrosati B. Conducting polymers: new electrochromic materials for advanced optical devices. Mol Cryst Liq Cryst. 1993;229(1):97-109.

21. Argun AA, Cirpan A, Reynolds JR. The first truly all-polymer electrochromic devices. Adv Mater. 2003;15(16):1338-41.

22. Sapp SA, Sotzing GA, Reddinger JL, Reynolds JR. Rapid switching solid state electrochromic devices based on complementary conducting polymer films. Adv Mater. 1996;8(10):808-11.

23. Heuer HW, Wehrmann R, Kirchmeyer S. Electrochromic window based on conducting poly (3,4-ethylenedioxythiophene) - poly (styrene sulfonate). Adv Funct Mater. 2002;12(2):89-94.

24. Hyodo K. Electrochromism of conducting polymers. Electrochim Acta. 1994:39(2):265-72.

25. Brooke R, Mitraka E, Sardar S, Sandberg M, Sawatdee A, Berggren M, et al. Infrared electrochromic conducting polymer devices. J Mater Chem C. 2017;5(23):5824-30.

26. Zozoulenko I, Singh A, Singh SK, Gueskine V, Crispin X, Berggren M. Polarons, bipolarons, and absorption spectroscopy of PEDOT. ACS Appl Polym Mater. 2019;1(1):83-94.

27. Wen Y, Xu J. Scientific importance of water-processable PEDOT-PSS and preparation, challenge and new application in sensors of its film electrode: a review. J Polym Sci A. 2017;55(7):1121-50.

28. Massonnet N, Carella A, Jaudouin O, Rannou P, Laval G, Celle C, et al. Improvement of the Seebeck coefficient of PEDOT: PSS by chemical reduction combined with a novel method for its transfer using freestanding thin films. J Mater Chem C. 2014:2(7):1278-83.

29. Wang J, Cai K, Shen S. Enhanced thermoelectric properties of poly (3,4-ethylenedioxythiophene) thin films treated with $\mathrm{H}_{2} \mathrm{SO}_{4}$. Org Electron. 2014;15(11):3087-95

30. Bubnova O, Khan ZU, Malti A, Braun S, Fahlman M, Berggren M, et al. Optimization of the thermoelectric figure of merit in the conducting polymer poly(3,4-ethylenedioxythiophene). Nat Mater. 2011;10(6):429-33.
31. Maddison D, Unsworth J, Roberts R. Electrical conductivity and thermoelectric power of polypyrrole with different doping levels. Synth Met. 1988:26(1):99-108.

32. Dubey N, Leclerc M. Conducting polymers: efficient thermoelectric materials. J Polym Sci B. 2011;49(7):467-75.

33. Twaha S, Zhu J, Yan Y, Li B. A comprehensive review of thermoelectric technology: materials, applications, modelling and performance improvement. Renew Sust Energ Rev. 2016;65:698-726.

34. Petsagkourakis I, Kim N, Tybrandt K, Zozoulenko I, Crispin X. Poly(3,4ethylenedioxythiophene): chemical synthesis, transport properties, and thermoelectric devices. Adv Electron Mater. 2019. https://doi. org/10.1002/aelm.201800918.

35. Myers R. Chemical oxidative polymerization as a synthetic route to electrically conducting polypyrroles. JEM. 1986;15(2):61-9.

36. Kumar D, Sharma RC. Advances in conductive polymers. Euro Polym J. 1998;34(8):1053-60

37. Waltman RJ, Bargon J. Electrically conducting polymers: a review of the electropolymerization reaction, of the effects of chemical structure on polymer film properties, and of applications towards technology. Can J Chem. 1986;64(1):76-95.

38. Gurunathan K, Murugan AV, Marimuthu R, Mulik UP, Amalnerkar DP. Electrochemically synthesised conducting polymeric materials for applications towards technology in electronics, optoelectronics and energy storage devices. Mater Chem Phys. 1999;61(3):173-91.

39. Bhattacharyya D, Howden RM, Borrelli DC, Gleason KK. Vapor phase oxidative synthesis of conjugated polymers and applications. J Polym Sci B Polym Phys. 2012;50(19):1329-51.

40. Brooke R, Cottis P, Talemi P, Fabretto M, Murphy P, Evans D. Recent advances in the synthesis of conducting polymers from the vapour phase. Prog Mater Sci. 2017:86:127-46.

41. McQuade DT, Pullen AE, Swager TM. Conjugated polymer-based chemical sensors. Chem Rev. 2000;100(7):2537-74.

42. Zhang $G, L u B$, Wen $Y$, Lu L, Xu J. Facile fabrication of a cost-effective, water-soluble, and electrosynthesized poly (9-aminofluorene) fluorescent sensor for the selective and sensitive detection of Fe(III) and inorganic phosphates. Sensor Actuat B Chem. 2012;171:786-94.

43. Zhang $G$, Zhang $H$, Zhang J, Ding W, Xu J, Wen Y. Highly selective fluorescent sensor based on electrosynthesized oligo (1-pyreneboronic acid) enables ultra-trace analysis of $\mathrm{Cu}^{2+}$ in environment and agroproduct samples. Sensor Actuat B Chem. 2017:253:224-30.

44. Zou L, Wen Y, Zhang H, Chai J, Duan X, Shen L, et al. Highly sensitive fluorescent sensor based on electrosynthesized poly (Fmoc-L-serine) enables ultra-trace analysis of $\mathrm{Cr}_{2} \mathrm{O}_{7} 2^{-}$in water and agro-product samples. Sensor Actuat B Chem. 2018;277:394-400.

45. Jian N, Lin K, Guo B, Zhang G, Liu X, Zou L, et al. A reusable fluorescent sensor from electrosynthesized water-soluble oligo (1-pyrenesulfonic acid) for effective detection of $\mathrm{Fe}^{3+}$. New J Chem. 2018:42(24):19450-7.

46. Bidan G. Electroconducting conjugated polymers: new sensitive matrices to build up chemical or electrochemical sensors. A review. Sensor Actuat B Chem. 1992;6(1-3):45-56.

47. Bobacka J, Ivaska A, Lewenstam A. Potentiometric ion sensors based on conducting polymers. Electroanalysis. 2003;15(5-6):366-74.

48. Bobacka J. Conducting polymer-based solid-state ion-selective electrodes. Electroanalysis. 2006;18(1):7-18.

49. Faridbod F, Norouzi P, Dinarvand R, Ganjali M. Developments in the field of conducting and non-conducting polymer based potentiometric membrane sensors for ions over the past decade. Sensors. 2008:8(4):2331-412.

50. Miasik JJ, Hooper A, Tofield BC. Conducting polymer gas sensors. J Chem Soc Faraday Trans 1. 1986:82(4):1117-26.

51. Arshak K, Moore E, Lyons G, Harris J, Clifford S. A review of gas sensors employed in electronic nose applications. Sensor Rev. 2004;24(2):181-98.

52. Bai H, Shi G. Gas sensors based on conducting polymers. Sensors. 2007;7(3):267-307.

53. Janata J, Josowicz M. Conducting polymers in electronic chemical sensors. Nat Mater. 2003;2(1):19.

54. Le Berre V, Angely L, Simonet-Gueguen N, Simonet J. Electroactive charge-transfer polymers from anodic coupling of dibenzo-crown ethers Part II. Influence of the complexing properties of the polyether rings. J Electroanal Chem. 1986;206(1-2):115-30. 
55. Walton D, Hall C, Chyla A. Functional dopants in conducting polymers Synth Met. 1991;45(3):363-71.

56. Rodrígez L-MT, Billon M, Roget A, Bidan G. Electrosynthesis of a biotinylated polypyrrole film and study of the avidin recognition by QCM. J Electroanal Chem. 2002;523(1-2):70-8.

57. Ho-Hoang A, Fache F, Boiteux G, Lemaire M. Synthesis and electrochemical characterization of functionalized 3-substituted polypyrroles. Synth Met. 1994;62(3):277-80.

58. Dong $S$, Sun Z, Lu Z. A new kind of chemical sensor based on a conducting polymer film. ChemComm. 1988;15:993-5.

59. Cadogan A, Lewenstam A, Ivaska A. Anionic responses of electrochemically synthesized polypyrrole films. Talanta. 1992;39(6):617-20.

60. Bendikov AT, Harmon T. Long-lived solid state perchlorate ion selective sensor based on doped poly(3,4-ethylenedioxythiophene) (PEDOT) films. Anal Chim Acta. 2005;551(1-2):30-6.

61. Lindfors T, Ivaska A. pH sensitivity of polyaniline and its substituted derivatives. J Electroanal Chem. 2002;531(1):43-52.

62. Mohammad F. Specialty polymers: materials and applications. New Delhi: IK International Pvt Ltd; 2007.

63. Persaud KC. Polymers for chemical sensing. Mater Today. 2005;8(4):38-44

64. Wei B, Ouyang L, Liu J, Martin DC. Post-polymerization functionalization of poly (3,4-propylenedioxythiophene)(PProDOT) via thiol-ene "click" chemistry. J Mater Chem B. 2015;3(25):5028-34.

65. Chivers T. Electron-rich sulfur-nitrogen heterocycles. Acc Chem Res. 1984;17(5):166-71.

66. Roncali J, Garreau R, Delabouglise D, Garnier F, Lemaire M. Modification of the structure and electrochemical properties of poly(thiophene) by ether groups. J Chem Soc Commun. 1989;11:679-81.

67. Marsella MJ, Swager TM. Designing conducting polymer-based sensors: selective ionochromic response in crown ether-containing polythiophenes. J Am Chem Soc. 1993;115(25):12214-5.

68. Allcock HR, Olmeijer DL, O'Connor SJM. Cation complexation and conductivity in crown ether bearing polyphosphazenes. Macromolecules. 1998;31(3):753-9.

69. Oh M, Stern CL, Mirkin CA. Coordination polymers from silver(i) and bifunctional pyridyl ligands. Inorg Chem. 2005;44(8):2647-53.

70. Zhang Z, Nguyen HT, Miller SA. Polymer-metal-organic frameworks (polyMOFs) as water tolerant materials for selective carbon dioxide separations. J Am Chem Soc. 2016;138(3):920-5.

71. Chang MN, Yang XK, Chhetri P, Chen JD. Metal and ligand effects on the construction of divalent coordination polymers based on bis-pyridylbis-amide and polycarboxylate ligands. Polymers. 2017;9(12):691-723.

72. Vázquez-Arce A, Zaragoza-Galán G, Aguilar-Ortíz E, Morales-Espinoza EG, Rodríguez-Alba E, Rivera E. Luminescent polythiophenes-containing porphyrin units: synthesis, characterization, and optical properties. Des Monomers Polym. 2014;17(1):78-88.

73. Warren L, Walker J, Anderson D, Rhodes C, Buckley L. A study of conducting polymer morphology the effect of dopant anions upon order. J Electrochem Soc. 1989;136(8):2286-95.

74. Hao Q, Lei W, Xia X, Yan Z, Yang X, Lu L, et al. Exchange of counter anions in electropolymerized polyaniline films. Electrochim Acta. 2010;55(3):632-40.

75. Rudd S, Franco-Gonzalez JF, Kumar Singh S, Ullah Khan Z, Crispin X, Andreasen JW, et al. Charge transport and structure in semimetallic polymers. J Polym Sci B. 2018;56(1):97-104.

76. Rolland N, Franco-Gonzalez JF, Volpi R, Linares M, Zozoulenko IV. Understanding morphology-mobility dependence in PEDOT: Tos. Phys Rev Mater. 2018;2(4):045605.

77. Inganäs $\mathrm{O}$, Erlandsson R. Proton modification of conducting polypyrrole. J Phys Chem Solids. 1983;45(4):427-32.

78. Khan ZU, Bubnova O, Jafari MJ, Brooke R, Liu X, Gabrielsson R, et al. Acido-basic control of the thermoelectric properties of poly(3,4-ethylenedioxythiophene)tosylate (PEDOT-Tos) thin films. J Mater Chem C Mater. 2015;3(40):10616-23.

79. Massonnet N, Carella A, de Geyer A, Faure-Vincent J, Simonato J-P. Metallic behaviour of acid doped highly conductive polymers. Chem Sci. 2015;6(1):412-7.

80. Kaufman J, Kanazawa KK, Street G. Gravimetric electrochemical voltage spectroscopy: in situ mass measurements during electrochemical doping of the conducting polymer polypyrrole. Phys Rev Lett. 1984;53(26):2461.

81. Pei $\mathrm{Q}$, Inganäs $\mathrm{O}$. Electrochemical applications of the bending beam method. 1. Mass transport and volume changes in polypyrrole during redox. J Phys Chem. 1992;96(25):10507-14.

82. Latonen RM, Akieh MN, Vavra K, Bobacka J, Ivaska A. Ion exchange behavior of polypyrrole doped with large anions in electrolytes containing mono-and divalent Mmetal ions. Electroanalysis. 2013:25(4):991-1004.

83. Stavrinidou E, Leleux P, Rajaona H, Khodagholy D, Rivnay J, Lindau M, et al. Direct measurement of ion mobility in a conducting polymer. Adv Mater. 2013;25(32):4488-93.

84. Pruneanu S, Csahok E, Kertesz V, Inzelt G. Electrochemical quartz crystal microbalance study of the influence of the solution composition on the behaviour of poly (aniline) electrodes. Electrochim Acta. 1998:43(16-17):2305-23.

85. Modarresi M, Franco-Gonzalez JF, Zozoulenko IV. Morphology and ion diffusion in PEDOT: Tos. A coarse grained molecular dynamics simulation. Phys Chem Chem Phys. 2018;20(25):17188-98.

86. Inzelt G. Redox transformations and transport processes. Conducting polymers: Springer; 2012. p. 191-244.

87. Ariga K, Hill JP, Endo H. Developments in molecular recognition and sensing at interfaces. Int J Mol Sci. 2007;8(8):864-83.

88. Thuau D, Ducrot P-H, Poulin P, Dufour I, Ayela C. Integrated electromechanical transduction schemes for polymer MEMS sensors. Micromachines. 2018;9(5):197.

89. Konstantin N. Ion-selective electrodes. In: Carpenter B, Ceroni P, Kirchner B, Koskinen A, Landfester K, Leszczynski J, et al., editors. St. Petersburg: Springer; 2013.

90. Buck RP. Ion selective electrodes. Anal Chem. 1976;48(5):23-39.

91. Kang Y-J, Lutz C, Hong S-A, Sung D-Y, Lee J-S, Shin J-H, et al. Development of a fluoride-selective electrode based on scandium(III) octaethylporphyrin in a plasticized polymeric membrane. Bull Korean Chem Soc. 2010;31(6):1601-8.

92. Philippe BLDC. Ion-selective electrodes with ionophore-doped sensing membranes. Supramol Chem Mol Nanomater. 2012;3:2539-76.

93. Shamsipur M, Rouhani S, Ganjali MR. A bromide ion-selective polymeric membrane electrode based on a benzo-derivative xanthenium bromide salt. Anal Chim Acta. 2000;418:197-203.

94. Wroblewski W, Wojciechowski K, Dybko A, Bianca H, et al. Uranyl salophenes as ionophores for phosphate-selective electrodes. Sensor Actuat. 2003;68:3113-318.

95. Vlascici D, Pruneanu S, Olenic L, Pogacean F, Ostafe V, Chiriac V, et al. Manganese(III) porphyrin-based potentiometric sensors for diclofenac assay in pharmaceutical preparations. Sensors (Basel). 2010;10(10):8850-64

96. Maria JBAC, Ibrahim HA. Tripodal ionophore with sulfate recognition properties for anion-selective electrodes. Anal Chem. 2000;72(21):5295-9.

97. La M, Hao Y, Wang Z, Han GC, Qu L. Selective and sensitive detection of cyanide based on the displacement strategy using a water-soluble fluorescent probe. J Anal Methods Chem. 2016;2016:1462013.

98. Sokalski T, Paradowski D, Ostaszewska J. Observations on the behaviour of some trifluoroacetophenone derivatives as neutral carriers for carbonate ion-selective electrodes. Analyst. 1996;21:133-8.

99. Karimi MA, Mashhadizadeh MH, Mazloum-Ardakani M, Rahavian F. Application of $\mathrm{H}$-point standard addition method and multivariate calibration methods to the simultaneous kinetic-potentiometric determination of cerium(IV) and dichoromate. Am J Anal Chem. 2010;01 (02):73-82.

100. Allen JR. pH electrodes, ion-selective electrodes, and oxygen sensors: electrochemical sensors used in the medical field. Lab Med. 2003;34(7):544-7.

101. Vázquez M, Bobacka J, Ivaska A. Potentiometric sensors for Ag + based on poly(3-octylthiophene) (РОT). J Solid State Electrochem. 2005:9(12):865-73.

102. Bobacka J, Vazquez M, Sundfors F, Mikhelson K, Lewenstam A, Ivaska A. Procedure 4 Determination of $\mathrm{Ca}(\mathrm{II})$ in wood pulp using a calciumselective electrode with poly (3,4-ethylenedioxythiophene) as ion-toelectron transducer. Compr Anal Chem. 2007;49:e25-8. 
103. Arroyo J, Akieh-Pirkanniemi M, Lisak G, Latonen R-M, Bobacka J. Electrochemically controlled transport of anions across polypyrrole-based membranes. J Membr Sci. 2019;581:50-7.

104. Bomar E, Owens G, Murray G. Nitrate ion selective electrode based on ion imprinted poly(N-methylpyrrole). Chemosensors. 2017;5(1):2.

105. Hyodo K, MacDiarmid AG. Effect of sulphate ion on the electrochemical polymerization of pyrrole and N-methylpyrrole. Synth Met. 1985;11:167-76.

106. Rudd S, Dalton M, Buss P, Treijs A, Portmann M, Ktoris N, et al. Selective uptake and sensing of nitrate in poly(3,4-ethylenedioxythiophene). Sc Rep. 2017;7(1):16581.
107. Rudd S, Desroches P, Switalska E, Gardner E, Dalton M, Buss P, et al. Relationship between structure/properties of vapour deposited PEDOT and sensitivity to passive nitrate doping. Sensor Actuat B Chem. 2019;281:582-7.

\section{Publisher's Note}

Springer Nature remains neutral with regard to jurisdictional claims in published maps and institutional affiliations.
Ready to submit your research? Choose BMC and benefit from:

- fast, convenient online submission

- thorough peer review by experienced researchers in your field

- rapid publication on acceptance

- support for research data, including large and complex data types

- gold Open Access which fosters wider collaboration and increased citations

- maximum visibility for your research: over $100 \mathrm{M}$ website views per year

At BMC, research is always in progress.

Learn more biomedcentral.com/submissions 